\title{
La problématique de l'ethnophilosophie dans la pensée de Marcien Towa
}

\section{Samba Diakité}

\section{CpenEdition}

Journals

Édition électronique

URL : https://journals.openedition.org/leportique/1381

DOI : 10.4000/leportique.1381

ISSN : $1777-5280$

\section{Éditeur}

Association "Les Amis du Portique"

\section{Référence électronique}

Samba Diakité, « La problématique de l'ethnophilosophie dans la pensée de Marcien Towa », Le Portique [En ligne], e-Portique, mis en ligne le 07 décembre 2007, consulté le 10 septembre 2022. URL http://journals.openedition.org/leportique/1381 ; DOI : https://doi.org/10.4000/leportique.1381

Ce document a été généré automatiquement le 10 septembre 2022

Tous droits réservés 


\title{
La problématique de l'ethnophilosophie dans la pensée de Marcien Towa
}

\author{
Samba Diakité
}

1 Frustrés et déçus des tracasseries de la colonisation, les intellectuels Africains présentent les normes d'une action possible face à la domination de l'idéologie coloniale. Ils affirment le droit pour les Africains à l'indépendance totale, à un nouveau style de pensées, de paroles et de vie. Cela se manifeste par l'exhumation de la culture africaine. A partir des tableaux d'occurrence et d'opportunité, d'apparition de l'Autre, le moment était enfin arrivé qu'une philosophie dite africaine puisse s'énoncer et ce, d'autant plus que déjà dans les domaines de l'art, de la littérature (entendue comme poésie et romans), des hommes Noirs avaient fait leurs preuves. Il manquait en quelque sorte la sanction philosophique à cette reconnaissance de la place du Noir ou de l'Africain dans le monde. Mais que vaut cette philosophie ? Pourquoi Marcien Towa la considère-t-il comme une ethnophilosophie ? Est-elle foncièrement dissociable de la philosophie européenne ? L'ethnophilosophie est-elle une philosophie africaine spécifique?

Dévoilement d'un concept

Dans les années 40, Placide Tempels, missionnaire du Katanga, va écrire une œuvre intitulée La philosophie bantoue. Mais ce livre, bien qu'il soit apprécié par des hommes de culture et des philosophes célèbres tels que Gabriel Marcel, Gaston Bachelard, Louis Lavelle, a été aussi sévèrement critiqué par des Africains comme Césaire, Mudimbé, Eboussi Boulaga, Hountondji, N'joh Mouellé, Marcien Towa,etc.

En effet, pour Marcien Towa, le but de Tempels n'est pas un but philosophique, mais un but hautement religieux. Car il tenait à trouver une nouvelle méthode de christianisation et de colonisation. Ainsi, la philosophie bantoue s'inscrivait-elle dans une tradition d'évangélisation, de domination économique, politique et culturelle de l'Afrique. 
4 Tempels ne cache pas son jeu, car il veut seulement inviter à respecter le Noir comme une personne ; et pour arriver à cette fin, il dresse le tableau d'une " philosophie bantoue » comme pour dire que les bantou ont déjà une philosophie, qu'ils pensent et qu'ils pourraient être de bons chrétiens s'ils faisaient un petit effort. Pour Towa, Tempels qui n'est pas philosophe, se montre soucieux d'une recherche philosophique pour mieux enseigner le message du Christ et il se dit que ce message serait mieux saisi s'il prenait en compte la logique bantoue. Par conséquent, il propose de renoncer à la logique pour épouser la manière de sentir et de voir du Noir, c'est-à-dire parler son langage, comprendre ses aspirations, rentrer en dialogue intime avec lui. Tempels semble le dire en ces termes :

" Nous ne prétendons pas que les bantous soient à même de nous présenter un traité de philosophie, exposé dans un vocabulaire adéquat (...) C'est nous qui pourrons leur dire, d'une façon précise, quel est le contenu de leur conception des êtres, de telle façon qu'ils se reconnaissent dans nos paroles, et acquiescent en disant : " tu nous a compris complètement, tu "sais" à la manière dont nous "savons"."

On comprendra alors pourquoi Tempels dénonce le système colonial qui nie tout droit aux Noirs. Ainsi va-t-il revendiquer le droit à la société, à l'éducation, à la vie, le droit à des conditions économiques et administratives décentes. Tempels sera ainsi emmené à dénoncer les exactions coloniales et, tout en les dénonçant, il dira que le Bantou a aussi une philosophie autant que les Occidentaux «dans la mesure où la philosophie était considérée comme l'attribut essentiel et indispensable d'une humanité véritable.»

C'est pourquoi, Marcien Towa pense que la notion de philosophie de Tempels, renvoie ici à la notion plus générale de philosophie primitive car «au lieu d'adopter à leur endroit l'attitude de détachement scientifique, les auteurs en quête d'une philosophie africaine spécifique leur confèrent une valeur normative relativement à la vérité ou à l'action». C'est à juste titre que Towa qualifie la pensée de Tempels d'Ethnophilosophie, car pour Tempels,

« le ressort et la fin de tout effort bantou ne peut être que l'intensification de la force vitale, sauvegarder ou augmenter la force vitale, voilà la clé et le sens profond de tous leurs usages. C'est l'idéal qui anime la vie du "muntu", c'est la seule réalité qui peut mouvoir le "muntu", c'est la seule cause pour laquelle il se trouve prêt à souffrir et à se sacrifier.»

7 Il faut signaler que ce concept, s'il est écrit en deux mots (ethno-philosophie) par Hountondji et en un mot par Marcien Towa, a d'abord été utilisé bien avant Towa et Hountondji, par Kwame N'krumah qui s'était inscrit à une thèse de Doctorat qu'il ne terminera jamais à l'Université Abraham Lincoln, aux Etats - Unis d'Amérique. Cette thèse de Doctorat avait pour sujet : «Esprit et pensée dans une société sans écriture, étude ethno - philosophique avec un examen particulier du cas du peuple akan de la Côte de l'Or.» En utilisant donc le terme d' " ethnophilosophie ", Marcien Towa veut montrer que l'ethnophilosophie n'est rien d'autre qu'un mouvement de réaction, tout comme la Négritude sa devancière. Pour lui, les défenseurs de l'ethnophilosophie africaine comme systèmes et philosophie des valeurs culturelles du Monde Noir, la présentent sous la forme d'une réalité transcendante par rapport à toutes les conditions matérielles et contingentes d'existence. L'Ethnophilosophie semble prendre pour de la philosophie, l'intégralité de la culture (mythes, contes, proverbes, magies, cosmogonies et sagesse, etc.). Elle serait la philosophie des systèmes de pensées collectifs, des traditions de pensée ou des visions du monde. Or, les valeurs culturelles 
et philosophiques du Monde Noir n'ont pas été et ne pouvaient pas être des productions sui-generis; elles sont, aujourd'hui encore, l'expression des conditions d'existence déterminées à la base. Elles n'ont rien des valeurs éternelles qui se tiendraient véritablement dans une fière indépendance, telles les idées platoniciennes dans leur environnement mystique. Dès lors,

" le problème est de saisir le lien qui assure leur cohésion, leur structure d'ensemble. Ce lien général, nous dit Towa, cette structure d'ensemble ne serait rien d'autre que la philosophie négro-africaine dans sa spécificité. En réalité, cette interprétation des données ethnologiques n'a pas pour objet d'établir l'existence d'une philosophie négro-africaine. Le résultat, but essentiel de l'entreprise, est obtenu dès l'instant où le concept de philosophie est élargi jusqu'à coïncider avec celui de la culture. Car l'ethnologie ou l'anthropologie culturelle a déjà établi que toute société humaine a une culture. Donner à la philosophie la même extension que la culture revient donc à poser aussi l'universalité de la philosophie. La philosophie, c'est le sens général de l'être - dans le monde de toute société».

8 Ainsi, remarquons-nous que Towa pense que les ethnophilosophes, pour se faire connaître de l'Occident, s'évertuent à construire en forme de philosophie, les matériaux de l'ethnologie traditionnelle. Towa ne se borne pas à dénoncer le caractère tautologique et mystificateur de cette démarche. Il la critique pour en montrer les limites et les conséquences néfastes. En effet, selon lui, si les travaux des ethnophilosophes permettent de saisir le monde négro-africain dans son unité, comme unité de civilisation irréductible à d'autres, ainsi que les traits caractéristiques, originaux et constants qui composent le type négro-africain et le système de "valeurs » qui est le sien, il semble difficile de les considérer comme des œuvres philosophiques. Tout en produisant un fond commun de concepts, de notions et de systèmes, une telle pratique est liée à une regrettable distinction entre un avoir et un être. D'ailleurs, cette philosophie qui insiste sur l'originalité et la revalorisation des cultures africaines, cette théorie en quête d'une «philosophie bantoue», d'une philosophie originale et authentiquement africaine, différente de toute philosophie européenne, cet humanisme qui sera la revendication fondamentale de la théorie politique de l'identité, a été perçu par Marcien Towa comme un obstacle à l'avènement de la révolution africaine. Les travaux de Tempels, Kagamé et autre Fouda, ne sont pas de la philosophie, mais de l'ethnophilosophie.

«Leur façon de procéder n'est ni purement philosophique, ni purement ethnologique, mais ethnophilosophique. L'ethnophilosophie expose objectivement les croyances, les mythes, les rituels, puis brusquement, cet exposé objectif se mue en profession de foi métaphysique, sans se soucier ni de réfuter la philosophie occidentale, ni de fonder en raison son adhésion à la pensée africaine. De la sorte, l'ethnophilosophie trahit à la foi l'ethnologie et la philosophie.»

9 Or, l'on sait que l'ethnologie est une discipline issue des sciences humaines et qui s'inscrit dans une visée unitaire. Elle veut étudier une société qui semble se définir par son unicité de langues, d'espace et de culture. Pour Marcien Towa,

" l'ethnologie, décrit, expose, explique mais ne s'engage pas (du moins pas ouvertement) quant au bien fondé de ce qui est ainsi décrit, expliqué. Elle trahit aussi la philosophie parce que la pierre de touche qui lui permet d'opérer un choix entre les diverses opinions est avant tout l'appartenance ou la non appartenance à la tradition africaine, alors qu'un exposé philosophique est toujours une argumentation, une démonstration ou une réfutation. Ce qu'un philosophe retient et propose est toujours du moins en droit, la conclusion d'un débat contradictoire, c'est-à-dire d'un examen critique et absolument libre ». 

sein de la littérature philosophique africaine.Si l'ethnophilosophie est une tentation permanente de la philosophie, si les ethnologues ont toujours cédé à l'envie de théoriser et de tirer des conclusions hâtives de leur philosophie, de leur pratique, cela ne veut pas dire qu'ils sont des philosophes. À dire vrai, il n'y a pas et il ne saurait y avoir de philosophie bantoue ou bantou, rwandaise, dioula, akan, européenne ou américaine si l'on entend par là la présence d'une pensée hypothétique homogène populaire à laquelle tous les bantu, rwandais ou dioula, etc. auraient adhéré massivement et unanimement. Cela enferme dans le particularisme, le culturel et le traditionnel. La notion de philosophie collective est aberrante. A penser le contraire correspondrait à la manifestation d'un manque à penser individuel à l'égard de ces peuples et de ces civilisations. Il n'y a pas une philosophie africaine commune et immuable et spécifique une fois pour toutes. Dès lors, il faut chercher la philosophie, disons la philosophie africaine, non seulement ailleurs, mais autrement.

Philosophie et ethnophilosophie : des différences foncières

La réflexion philosophique est un besoin parmi les besoins et comme telle, elle serait un effort de mise en ordre du monde. Le philosophe cherche le vrai mais le vrai n'est pas encore le réel, car le vrai reste enfermé dans le cadre du jugement. Par conséquent,

« la question de savoir si nous avons ou non une philosophie, doit donc être résolument subordonnée à l'examen impartial et au jugement objectif de la valeur intrinsèque de la philosophie, au sens européen du terme et au rôle qu'elle est susceptible de jouer relativement à notre dessein fondamental. »

Pour Marcien Towa, l'ethnophilosophie est une anti-philosophie, car le philosophe doit s'inspirer de la science ou, mieux, des sciences. Or, les sciences ne s'éclairent que par des théories qui dépassent les sens. Ces théories ne sont pas celles qu'imaginent les savants pour faire progresser leurs disciplines. Elles sont pour le philosophe une recherche de principes. Pour Towa, toute connaissance doit impérativement passer sous le crible de la raison. Et si la raison peut être à la fois le sujet et l'objet de la critique, c'est qu'elle est ce pouvoir spécifique et parfaitement original que possède la pensée d'opposer à ce qui est ce qui doit être. Elle doit imprimer à la pure et simple existence, ce qu'elle ne crée pas et que seule l'expérience peut lui révéler. Le sceau d'une nécessité et d'une universalité doit exprimer son exigence normative. L'acte propre de la pensée est le jugement qui décide de toute chose comme d'un cas relevant d'une règle. L'objet propre de la philosophie, comme connaissance de la raison humaine émane des conditions nécessaires à l'exercice légitime de sa propre normativité. Or, dit Towa,

" la philosophie populaire recherche moins la pénétration intellectuelle que l'édification par l'enthousiasme enflammé pour le beau, le sacré ou la religion. Elle croit trouver dans ce zèle brûlant un raccourci vers le vrai, lui épargnant de suivre le long chemin de culture philosophique, le mouvement riche et profond à travers lequel seul l'esprit parvient au savoir. Le résultat décevant en est en fait la présomption d'idées toutes faites qu'on n'estime pas utile de soumettre à la discussion (...) Le recours au sens commun, à la pureté de la conscience ou à l'innocence du cœur comme fondement ultime des "vérités avancées" par la philosophie populaire ruine en fait toute possibilité de dialogue et d'accord entre les hommes. »

13 La réflexion qui caractérise la philosophie doit prendre la forme d'un reflux de la pensée sur ses propres sources vives qui lui permet de se ressaisir comme l'origine du 
sens qu'elle confère à ses objets et à ses œuvres. Loin que les réponses à ses questions soient déjà quelque part dans l'au-delà d'une transcendance plus ou moins inaccessible, elles ne se découvrent que progressivement dans leurs liens, aux problèmes que l'esprit peut et doit se proposer, comme autant de tâches à accomplir. Assurément, comme toute connaissance digne de ce nom, la philosophie vise bien cette valeur de vérité qui se définit par l'accord de la pensée avec son objet. Mais son objet à elle, c'est le critère de cette vérité qui ne qualifie pas seulement les solutions, mais les problèmes euxmêmes. Le lieu qui lui revient en propre ne se situe pas dans le ciel. L'homme ne peut s'exalter jusqu'à des visions supraterrestres car nous dit Towa :

"La solution de nos problèmes ne se trouve pas au ciel, entre les mains des dieux ou des chefs charismatiques, des hommes providentiels et autres sauveurs. Attendre d'eux la réponse à nos interrogations théoriques et à nos hésitations pratiques, c'est fuir le nécessaire effort de réflexion, de pensée personnelle par la discussion et la recherche méthodiques.» Or, l'ethnophilosophie n'est rien d'autre que le témoignage des sociétés tribales, ethnocentriques. Elle se ramènerait à la problématique centrale de la pensée tribale. La problématique de l'ethnophilosophie n'est que la pratique victorieuse d'une pensée douteuse et mythique. Or,

" la mentalité mythique érige directement un comportement individuel en norme universel de comportement, une opinion individuelle en vérité universelle, du seul fait qu'il s'agit du comportement de la volonté ou des déclarations d'une individualité, homme ou dieu posé comme exemplaire (...) Ce qui caractérise donc essentiellement un esprit mythique, c'est son inaptitude ou son renoncement à penser, à réfléchir d'une manière personnelle et autonome.» Selon Towa, le mythe prôné par l'ethnophilosophie ne peut être de la philosophie, car le mythe est une invitation à la divagation émerveillée de l'esprit à travers le temps et l'espace. La philosophie, quant à elle, refuse de livrer les hommes à la tyrannie, ennemie de la liberté et de la pensée. Son rôle est seulement de penser les croyances mythologiques tout en les pesant et les soupesant sous le regard vigilant de l'esprit. Toute l'évolution ultérieure de l'ethnophilosophie traduit les ruses inopérantes d'une civilisation incapable de surmonter les apories soulevées par son propre développement. Idéologiquement, l'effort de l'ethnophilosophie consiste à empêcher chez le dominé toute prise de conscience de sa condition réelle de dominé et d'esclave en le dépréciant à l'histoire. Selon Marcien Towa, à l'heure où se creuse partout sur notre continent le fossé entre oppresseurs et opprimés, à l'heure où se radicalisent les divergences politiques, l'ethnophilosophie prétend que nous avons toujours été, que nous sommes et seront toujours unanimes. Et pourtant, puisqu'en ce domaine, elle n'a affaire qu'à elle-même, elle devrait résoudre les problèmes qu'elle se pose et qui ne lui sont pas imposés par la variété infinie des objets, la richesse inépuisable des choses - qu'elle en soit définitivement incapable pourrait nous faire perdre toute confiance en elle et engendrerait le scepticisme. C'est un fait, cependant, que loin de progresser d'un pas assuré qu'on a vu prendre à la philosophie, l'ethnophilosophie n'a cessé au contraire d'offrir le spectacle humiliant pour la raison humaine d'un perpétuel champ de bataille où les ethnophilosophes s'affrontent depuis des années en des combats sans issue. Livrée à elle, en ethnophilosophie, la raison devient statique : les conclusions de ses raisonnements sont contestées, sa législation devient antinomique et se montre incapable de trancher entre les thèses qui s'excluent. Ainsi le succès qu'elle connaît en Afrique et qu'attestent les progrès incessants de la philosophie et de la science, conduit 
plutôt à soupçonner que sa singulière destinée en philosophie, où elle ne peut pas plus esquiver les questions que leur donner une réponse, provient d'un malentendu, d'une méprise que l'on doit pouvoir dissiper. Il y a une énigme à déchiffrer et sans doute une nouvelle route à trouver. Si l'on parvient à savoir comment on parvient à la notion de philosophie et ce qui caractérise la philosophie, on disposera du même coup d'un critère permettant de décider de l'aptitude de l'ethnophilosophie à y parvenir et de trouver la voie qu'elle devra suivre à cette fin.La solution de ce problème présente un caractère préjudiciel en ce sens qu'il a été formulé parce qu'on voulait savoir si l'ethnophilosophie est possible comme philosophie ; ce qui a conduit à s'enquérir d'abord des conditions qui rendent possible les philosophies existantes en y retrouvant la manifestation du pouvoir de connaître qui est propre à l'homme. La philosophie s'accorde à la critique et celle ci est d'abord cette méthode nouvelle, voire une science des conditions qui permettent à la raison de constituer la science et du coup lui permettront aussi de constituer les éléments de l'ethnophilosophie en éléments d'études philosophiques. Ce caractère préliminaire lui assigne une place bien à part : elle est la connaissance que l'ethnologie prend d'elle-même. Pouvoir de connaître, il faut qu'elle sache dans quelle mesure elle peut y parvenir ; puissance législatrice selon la formule de Kant, il faut que l'ethnophilosophie se fasse comparaitre à son propre tribunal où seront reconnus ses droits, condamnées ses compétitions abusives, bref où il sera décidé des limites à l'intérieur desquelles elle peut légitimement faire usage de ses principes. La philosophie elle-même doit viser à désigner cette entreprise qui se propose non pas l'extension des connaissances ethnophilosophiques, mais leur justification, selon un jugement qui décide de leurs conditions de validité. L'ethnophilosophie proposera ainsi l'idée d'une philosophie nouvelle pour souligner qu'elle n'entend pas être une connaissance des cultures et des valeurs africaines, mais seulement de leurs concepts dans la mesure où cela est possible a priori, bref la philosophie de l'usage légitime des éléments ethnophilosophiques. Mais la critique se proposant avant tout de frayer à l'ambition ethnophilosophique de la culture africaine, cette voie royale de la "connaissance" africaine que celle-ci a déjà su trouver pour son entreprise philosophique, elle doit également pouvoir se présenter comme un traité de méthode. Tout naturellement, l'acte de naissance des philosophies déjà constituées, prend la valeur d'un exemple et d'un modèle pour l'ethnophilosophie. D'ailleurs, comme le dit Dibi Augustin,

«Si le peuple africain revendique un trait tel que la solidarité ou l'hospitalité comme une qualité qui le déterminerait en propre, immuable et irréductible au temps, ce trait devient une hypostase, quelque chose de solide, en tendant à se suffire à lui-même, ne parvient plus à rendre raison de ce pourquoi l'on est solidaire. Ainsi cristallisée, parce qu'il n'est plus tenu compte de l'articulation inévitable de l'ensemble des questions humaines et des déplacements subis par cette articulation dans l'histoire, la solidarité n'est plus vécue par l'individu que comme une simple couverture en se faisant intérieurement violence, puisque l'individu agira pour se rendre extérieurement conforme à une image qui ne vient pas de sa volonté intime (...) Dans cette fermeture sur soi du singulier jusqu'à l'extinction de la visée d'intentionnalité - qui pourtant lui rend raison et à son propre obscurcissement, comment le désir de reconnaissance pourrait il avoir la chance d'aboutir?»

Or, il est décisif que l'ethnophilosophie revêtît la forme d'une révolution, d'une conversion. L'ethnophilosophie rentrera dans la voie de la philosophie lorsqu'elle aura cessé d'être tenue en lisière par l'ethnologie et qu'elle aura entrepris de la soumettre à ses propres exigences, car elle ne peut pleinement saisir que les objets produits par son 
initiative. Il faut, cependant, reconnaître que l'intention des ethnophilosophes était bonne, car leur " Science" était une sorte de "garde - fou de la civilisation africaine ", défenseur inébranlable de la culture africaine en face des aberrations des sociétés industrielles. Mais, en fait, Towa croit que les Africains à un moment donné ont pris conscience de leur état d'êtres assujettis, car on avait cru à tort que seule l'intuition caractérisait l'esprit africain. L'Africain se trouverait dans un rapport immédiat avec son objet et celui-ci est tel que le sujet est englouti dans la substantialité, si elle n'est pas enfermée dans les ténèbres de la nuit. L'esprit africain, avait - on dit, n'arrive pas à se défaire de l'unité compacte des choses et de l'existence pour s'affirmer dans sa liberté subjective. L'homme Africain en tant que sujet, n'aurait malheureusement pas encore produit de lui-même l'objet universel afin de parvenir à l'histoire universelle selon la formule de Hegel. L'objet quant à lui, n'aurait pas encore eu sa seconde naissance dans le sujet, comme nous l'avons déjà dit. Son mode d'être spirituel ne serait pas encore objet de représentation, mais se caractériserait formellement par l'immédiateté. C'est pourquoi, l'objet visé, ne serait rien d'autre qu'un sujet déterminé par l'immédiateté et d'une manière immédiate à l'instar du soleil naturel hegelien. Comme le soleil, il serait l'image de la fontaine sensible et somnambulique, non spirituelle et non dévouée, et apparaîtrait par conséquent comme un individu naturel. Fort de ces critiques, certains Africains vont s'appuyer sur les mythes, la littérature orale, la culture et autres proverbes pour affirmer l'existence d'une philosophie africaine propre, tout en posant la limitation et l'imperfection de tout être réel ou considéré comme tel. Ces auteurs Africains s'évertueront à poser en principe la nécessité d'un effort, d'une réflexion en commun, d'un échange de vues, d'un débat pour la détermination véritable des valeurs et des normes suprêmes. Mais ce qu'il y a de profondément anti-philosophique dans ces mythes et ces proverbes,

«c'est cette fermeture sur soi du cercle des connaissances. L'esprit philosophique est avant tout un esprit d'ouverture, de nécessaire dépassement des contingences, un esprit scientifique aussi pour finir.»

17 Par ailleurs, à force de s'interroger sur "l'homo-africanus", le type negro-africain, on risque de l'hypostasier et l'on finit par ne pas comprendre les réalités africaines du moment. Ne tenant donc pas compte de la différence philosophique, «l'ethnophilosophie telle qu'elle s'est pratiquée jusqu'ici n'a trop souvent été qu'une voie de facilité, faisant l'économie à la fois des techniques et des méthodes d'enquête ethnologique et de la discussion philosophique des idées et des valeurs mises en avant, et tout ceci, au nom de l'africanité!» C'est pourquoi, nous dit Towa, elle est un obstacle à l'avènement de la révolution africaine, tout comme la néo-bourgeoisie et la Négritude qui l'ont vu naître.

«Et en esquivant le débat philosophique sur les idées et les valeurs, il ne lui reste pour les imposer, que la voie d'un dogmatisme desséchant dans lequel la négritude entendue comme retour à nos sources culturelles dans la fierté retrouvée, est pervertie, au point de n'être plus qu'un avatar du «magister dixit». En raison d'un tel dogmatisme, les idées avancées par l'ethnophilosophie sont figées dès leur mise au jour et ne sont susceptibles d'aucun développement.»

18 Il est vrai, chaque société, chaque collectivité, organise en Afrique, comme partout ailleurs, ses activités et son comportement en vue de certains objectifs fondamentaux et de certaines valeurs essentielles, conformément à certaines normes, à certaines éthiques, s'imposant au respect de tous. Ces normes peuvent reposer sur des mythes, sur des récits sacrés qui ont pour héros des êtres plus ou moins surnaturels. Mais cela 
ne doit pas être traité de philosophique car la démarche philosophique se caractériserait selon Towa, par une liaison intime entre le souci de connaître rationnellement, méthodiquement, la réalité des choses et une bonne volonté de prendre appui sur ce savoir pour définir l'orientation profonde, absolue que doit adopter le comportement humain. Il est vrai, " avec le mythe, l'homme crée la première culture, humanise le monde, c'est-à-dire l'organise à une totalité directement ou indirectement anthropocentrique. Mais en définitive, l'univers mythique, parce qu'il se fonde sur l'autorité de la tradition, ennemi de toute critique et de tout examen libre et personnel, fige les adaptations réalisées une fois pour toutes en comportements purement répétitifs et rituels et condamne à l'immobilisme (...) En fait le mythe ne fait que reproduire la nature et l'instinct.» Aussi faut-il admettre que l'exploitation de notre passé, l'examen de notre culture traditionnelle obéit à un souci d'objectivité et de connaissance de soi. Il importe donc de nous connaître tels que nous sommes, tel que le passé, proche ou lointain, nous a façonnés, d'appréhender l'acquis de nos ancêtres en vérité, dans toute sa splendeur et sa diversité. Il ne doit être ni approuvé, ni défiguré, ni momifié pour être montré en spectacle. La culture peut être très stérile : c'est là une chose bien connue. Mais, nous disons aussi que la culture ne doit pas exister seulement dans la tête de l'homme mais aussi dans son cœur. En vérité, c'est la réalité de l'homme tout entière qui doit exprimer la culture ; et l'homme est essentiellement un être qui doit avoir le sens de l'ordre éthique et du droit.

19 Si tel ou tel individu peut être conforme au principe de la vérité et de la justice, il n'en va pas de même pour les peuples et les ethnies. Ici, le principe universel imprègne toutes les sphères particulières de la vie et la vie en tant que conscience culturelle pratique, est pénétrée par la vérité. Ce qui se réalise dans la constitution politique, les relations juridiques, l'ordre éthique en général ou l'art et la science ne sont rien d'autre que la manifestation même de la vérité et de l'ordre dans ces différentes sphères.

«Il est un fait dialectique que l'insistance unilatérale sur une détermination finit par se dissoudre en soi-même, puisque l'extrême, en ne connaissant plus de mesure, ne peut plus être qualifié : ce qui par suite, signifie pour lui la perte de tout. L'Afrique échoue à faire reconnaître à l'Europe sa particularité, parce que celle-ci, visée comme une chose, substantiellement, comme une détermination immuable qu'aucun regard venant de l'extérieur ne peut pénétrer, confesse de cette façon même qu'elle trouve seulement dans l'obscurité sa fidèle compagne et ne peut rien communiquer.»

Conclusion

En définitive, ce que Marcien Towa veut montrer à travers la critique de l'ethnophilosophie, c'est qu'il appartient maintenant à nous Africains, d'apporter à l'histoire, la foi et l'idée que le monde du Vouloir n'est pas seulement livré à l'Occident, qu'il n'appartient pas non plus au hasard. Nous n'avons pas d'autre but que d'éliminer le hasard. Nous devons chercher dans notre histoire, un but universel pour nous réaliser dans l'existence et développer nos propres personnalités. L'homme Africain doit s'efforcer de comprendre fidèlement l'histoire, d'appréhender le vrai car le vrai ne doit pas résider seulement dans la superficie sensible comme nous l'ont fait croire la Négritude et l'Ethnophilosophie. En toute chose, en particulier, dans tout ce qui est être scientifique et valorisé, la raison ne doit dormir, elle ne doit pas être titubante et ivre. Il faut user de la réflexion. 
Voir et analyser le monde rationnellement, méthodiquement, objectivement, c'est aussi être vu rationnellement, méthodiquement, objectivement, par le monde. Mais pour connaître l'universel, la rationalité, on doit apporter avec soi la raison. Pour reconnaître et jouir de la modernité, on doit apporter avec soi aussi la raison et les matériaux nécessaires pour être "moderne". Pour connaître la substance de la modernité, il faut l'aborder avec raison, prendre conscience de la raison, disons de sa propre raison pour transpercer l'apparence auréolée et bariolée des événements. Pour comprendre l'essentiel aujourd'hui, il nous faut d'abord éliminer l'inessentiel selon les buts que nous poursuivons dans la considération de notre continent. L'heure est maintenant venue pour nous de nous poser sérieusement cette question : Modernité ou Désespoir de la raison, quel choix pour l'Afrique ? Cette question est d'autant plus importante, qu'en Afrique aujourd'hui, d'énormes difficultés se posent à nous. C'est une mêlée bigarrée de problèmes qui nous emporte et dès qu'un problème disparaît, un autre aussitôt prend sa place. Et le côté négatif de ce spectacle du changement provoque assurément notre tristesse et notre angoisse. Il est déprimant et désespérant de savoir que tant de splendeur, tant de vitalité et d'harmonie que contenait l'Afrique, est en train de périr et que nous sommes en train de marcher au milieu des ruines dans les cendres du désespoir avec l'œil de la Raison. Quelle Raison ! Tout semble voué à l'échec, à la disparition. Rien ne demeure. Tout s'écroule et nous nous écroulons avec ce tout. Cependant, à cette catégorie du changement, à ce désespoir de la Raison, doit se rattacher aussitôt un nouvel aspect : l'espoir de la Raison, l'avènement de la "vraie" modernité. N'est- ce pas que c'est de la mort que renaît la vie ? Mais cette vie nouvelle ne doit pas être seulement pour l'Afrique, le rajeunissement d'un simple retour à la forme antérieure, elle doit être nécessairement une purification, une transformation, pour tout dire une Révolution Radicale.

\section{BIBLIOGRAPHIE}

Ouvrages de Marcien Towa

Towa (Marcien). L'idée d'une philosophie négro-africaine (Yaoundé, Clé, 1979),

Towa (Marcien), Essai sur la problématique philosophique dans l'Afrique actuelle, Yaoundé, Clé, 1975

Towa (Marcien), Essai sur la problématique philosophique dans l'Afrique actuelle, Yaoundé, Clé, 1981

Towa (Marcien),"Les conflits entre traditionalismes : recherche d'une solution" in Recherche, pédagogie et culture, $n^{\circ} 56$, Paris, AUDECAM, 1982.

Towa (Marcien).Léopold Sédar Senghor : négritude ou servitude (Yaoundé, Clé, coll. Points de vue, 1971)

Autres ouvrages

Adotévi (Stanislas Spéro Kodjo), Négritude et négrologues, Paris, Union Générale d’Editions, Coll. 10/18, 1972. 
Arendt (Hannah), La crise de la culture, traduction de Jacques Bontemps et Patrick Lévy, Paris, Gallimard, 1999.

Cioran, Histoire et utopie, Paris, Gallimard, 1960.

Diakité (Tidiane), L'Afrique est malade d'elle-même, Paris, Karthala, 1999.

Dibi (Augustin Kouadio), L’Afrique et son autre : la différence libérée, Abidjan, Stratéca Diffusion, 1994.

Diop (Cheikh Anta), Nations nègres et cultures, Paris, Présence Africaine, 1970, tomes I\&II

Diop (Cheikh Anta), Antériorité des civilisations nègres : mythe ou vérité historique?, Paris, Présence Africaine, 1980.

Hegel (Georg Wilhem Friedrich), Leçons sur l'histoire de la philosophie, traduction J. Gibelin, Tome I, Paris, Gallimard, 1954.

Hegel (Georg Wilhem Friedrich), La raison dans l'histoire, traduction Kostas Papaioannou, Paris, Union Générale d'édition,Coll.10/18, 1985.

Henri-Lévy (Bernard), La barbarie à visage humain, Paris, Grasset\& Fasquelle, 1977

Henri-Simon (Pierre), L'esprit et l'histoire, Paris, Petite Bibliothèque Payot, 1969,

Hountondji (Paulin Jidenu), Sur la "philosophie africaine". Critique de l'ethnophilosophie, Paris, Maspero, 1977.

Kane (Cheick Amidou), L'aventure ambiguë, Paris, Julliard, 1961.

Kouassigan (Guy Adjété), Afrique : Révolution ou diversité des possibles ? Paris, L'Harmattan, 1985.

Marcuse (Herbert), Eros et civilisation, Traduit en anglais par Jean- Guy Nény et Boris Frankel, Paris, Minuit, 1963.

Memmi (Albert), Le racisme, Paris, Gallimard, 1984.

Meister (Albert). L'Afrique peut-elle partir ?, Paris, Seuil, 1966.

Njoh-Mouelle (Ebenezer), Jalons II, L'Africanisme aujourd'hui, Yaoundé, Clé, 1975

Sebag (Lucien), Marxisme et structuralisme, Paris, Petite Bibliothèque Payot, 1964.

Serres (Michel), Le Tiers-Instruit, Paris, Gallimard, 1991.

Schroyer (Trent), Critique de la domination, traduit de l'américain par Jacques Debouzy, Paris, Payot, 1980.

Sine (Babacar), Le marxisme devant les sociétés africaines contemporaines, Paris, Présence Africaines, l'Herne, 1987, collection bibliothèque de philosophie d'esthétique, 1983.

Smet (A.J.), Philosophie africaine, (textes choisis I), (Kinshasa, PUZ,1975.

Smet (A.J.), Philosophie africaine (textes choisis II et bibliographie sélective), Kinshasa, PUZ, 1975.

Stephane (André), L'univers contestationnaire, Paris, Petite Bibliothèque Payot, 1969.

Tempels (R-P-Placide), La philosophie bantoue (Paris, Présence Africaine, 1961).

Verstraeten (P.), Révolution de mai et idéologie communiste in lutte de classes ou conflits de génération (Bruxelles, Cercle d'éducation populaire, 1969.

Revues et articles

Binda (N), "Comprendre Tempels" in Revue Pédagogie et culture, №58 (Paris, AUDECAM, 1982) 
Fabian (Jean.), "Philosophie bantoue : Placide Tempels et son œuvre vus dans une perspective historique" (Bruxelles, Centre de recherches et d'information sociologique, 1970)

Bollart (E.), "La philosophie bantoue selon le R.P. Placide Tempels" in Philosophie africaine, Textes choisis II, (Kinshasa, Puz, 1975)

Bouillon.- "Emprise discursive et domination" in Communication (Paris, Seuil, 1978), $\mathrm{N}^{\circ} 28$.

Boulaga (Fabien Eboussi, "Le bantou problématique" (Paris, Présence Africaine, 1968), N66

Boulaga (Eboussi Fabien), "Le bantou problématique" in Présence Africaine Nº69 P4-88, 1968.

Crahay (Frantz). "Le décollage conceptuel condition d'une philosophie bantoue“ in DIOGENE, $\mathrm{N}^{\circ}$ $52,1965)$.

Crahay (Frantz), "Le décollage conceptuel condition d'une philosophie bantoue" in Philosophie africaine (Kinshasa, PUZ, 1975).

Dailly (C.), "Vers une révolution de l'idéologie et de la littérature négro-africaine" In Revue de la littérature et d'esthétique négro-africaine, tome1, (Abidjan, NEA 1977)

Derrida (Jacques), "La pharmacie de Platon" in Tel Quel (N³2 Hiver 1968, pp 8).

De Sousberghe, "A propos de la philosophie bantoue" in Philosophie africaine, Textes choisis II ? (Kinshasa, Puz, 1975)

Hountondji (Jidenu Paulin).- "table ronde sur la philosophie africaine" in Recherche, Pédagogie et culture, $\mathrm{N}^{\circ} 56$, Janvier-Mars 1982

Hountondji (Jidenu Paulin), Que peut la philosophie? (Paris, Maspero, 1977).

Hountondji (Jidenu Paulin). " Occidentalisme, élitisme : réponse à deux critiques" in Recherche, Pédagogie et culture, ( ${ }^{\circ} 56$, Janvier-Mars 1982, PP.58-67).

Howlett.- "La philosophie en question" in Présence africaine, (N91,1974, PP. 14-25).

\section{RÉSUMÉS}

Philosophe Camerounais, Marcien Towa fait une critique sans complaisance de l'ethnophilosophie africaine qui, selon lui, est un sous-ensemble de l'ethnologie européenne. Il montre que la philosophie africaine, si elle existe, devrait se conformer à la philosophie au sens strict du terme. Il est maintenant nécessaire, pour la philosophie africaine, d'éliminer les obstacles à la révolution africaine afin que l'Afrique puisse amorcer véritablement son processus d'auto-libération et d'auto-émancipation. Mots-clés : Ethnophilosophie-Révolution-ColonisationPhilosophie-Valeur-Culture-Mythe-Critique

\section{AUTEUR}

\section{SAMBA DIAKITE}

Dr Samba DIAKITE est Enseignant-chercheur à l'Université de Bouaké (Côte d'Ivoire. BP 802 Anyama docteursamb@yahoo.fr Spécialiste en Philosophie Africaine l'UFR Communication - Milieu et Société, département de Philosophie, il est aussi Directeur des Etudes au CESTIA-2EP( Centre d'Enseignement Supérieur et des Technologies Internationales d'Abidjan -Ecole Entreprise Placement). Il a, à son actif, plusieurs travaux de recherches dont : « La formule incantatoire en Afrique : approche 
philosophique », in Le Korè, Revue Ivoirienne de Philosophie et de Culture, n³5 (Abidjan, Éditions Universitaires de Côte d'Ivoire (EDUCI), 2004). Côte d'Ivoire ; « La déréliction du langage dans le penser politique en Afrique », in Le Portique, 2005-eportique 1, http://leportique.revues.org/document 521. Html, France ; « La foi des éclaireurs ", in Le Korè, Revue Ivoirienne de Philosophie et de Culture, Nº36, (Abidjan, EDUCI, 2005) Côte d'Ivoire ; «Les enjeux de la guerre », in Les Cahiers du CERLESHS (Centre d'Études et de Recherche en Sciences Humaines et Sociales) $\mathrm{N}^{\circ} 23$ Ouagadougou, Presses Universitaires de Ouagadougou, 2005), Burkina Faso ; « L'Autre et sa langue : la langue du refus » in Contrepoint philosophique, www.contrepointphilosophique.ch « Marcien Towa entre deux cultures »in Le Korè, Revue Ivoirienne de Philosophie et de Culture, N³7, (Abidjan,EDUCI, 2006) Côte d'Ivoire. 